\title{
Translating pharmacogenomics discoveries into clinical practice: the role of curated databases
}

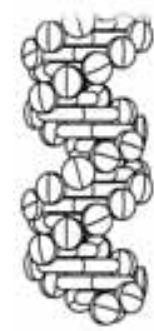

\section{Prakash M Nadarnit \& Mathieu Wiepert?}

${ }^{\dagger}$ Author for correspondence 'Yale U niversity School of M edicine,

New Haven,

CT 06511, USA

Fax: +1 203737 5708;

E-mail: Prakash.N adkarni

@yale.edu

${ }^{2} \mathrm{M}$ ayo $\mathrm{Clinic}$

Rochester,

MN 55905, USA

\author{
Systematic classific ation of \\ disea ses and adverse effects can \\ improve the value of electronic \\ pharmacogenomic information.
}

Pharmacogenomics-related genotype information is growing at a supralinear rate, and phenotyperelated information, as determined by computer simulations, in vitro experiments and clinical studies, is also growing. Even when phe notypic information is confirmed via clinical research, numerous barriers exist in translating these discoveries into clinical practice. We consider two of them here: the uncertainty regarding the practical relevance of research observations, and the translation of significant research findings into clinical practice and research through electronic information access. This form of access is critical because even the leading clinical pharmacologists cannot fully mentally retain today's large volume of drug-related information.

Clinical importance of individual pharmacogenomics studies

It has been suggested that HIV viral genotyping, which is part of routine patient management in the USA, can serve as a paradigm for the introduction of pharmacogenomics into patient care [1]. H owever, the important difference is that viral genotyping predicts drug resistance very accurately, in addition to helping to trace the transmission of specific viral strains through susceptible communities. In contrast, the detection of most human polymorphic gene variants (which, by definition, are common in individual populations) is often of less clear consequence: their effects are typically much less dramatic than those of the rare variants, such as the atypical butyrylcholinesterase gene, which instigated classical pharmacogenetics.

Even for well-characterized polymorphisms that are widely accepted to be clinically important, the variation in phenotypic end points such as metabolic ratios (M Rs) of non-metabolized to metabolized drugs are considerable. For example, in a study of 200 Iranian subjects phenotyped for cytochrome P450 (CYP)2D 6 polymorphisms with dextromethorphan, a 520-fold inter-individual $M R$ variation was observed across all subjects, with variation for poor metabolizers itself being 19.5-fold [2]. Even when the genotype and phenotype correlate with high statistical significance, their clinical importance is harder to assess. Thus, in a recent study of warfarin kinetics [3], multiple regression analysis of warfarin maintenance dose as a function of CYP2C 9 genotype, age, lean body weight, and concomitant treatment with warfarin metabolism inducers yiel ded a statistically significant $r^{2}$ (coefficient of determination) value of 0.37 . This number, however, implies that $63 \%$ of the experimental variance is unaccounted for.

Consequently, there are differences between the viewpoints of those generating the results and those who are expected to act on them. Despite recommendations that CYP2D 6 genotyping 'should be performed routinely as genotyping costs offset medical costs' [4], a recent Australasian survey of 629 individuals [5] found that only one center was routinely performing CYP2D 6 phenotyping (preparatory to perhexilene therapy), while genotyping and phenotyping tests for other CYP enzymes, $\mathrm{N}$-acetyltransferase 2 and dihydropyrimidine dehydrogenase, were effectively never undertaken for clinical purposes. This survey concluded that 'a poor evidence base, unestablished clinical relevance and, in the few cases with the strongest rationale, a slow translation to the clinical setting' were the causes of low clinical utilization of CYP2D 6 genotyping.

In the personalized medicine scenario of pharmacogenomics [101], it is assumed that stored patient data on critical gene variants, related to drug action or kinetics, will be accessed by both humans as well as software to improve clinical decision-making. However, as a recent article in this journal by M CLeod emphasizes [6], the challenging diseases of today involve complex biological pathways where multiple genes interact. D espite advances in the field of systems biology (also recently reviewed in this journal by Yengi [7]), the ability to accurately predict the composite effect of multiple gene variants in a patient, where some indicate favorable prognosis (if existing in isolation) while others indicate an unfavorable prognosis, does not currently exist. 
Translation of research into practice: status of current electronic databases Electronic databases allow clinicians to keep pace with information growth. The primary data source is still the biomedical literature: the impact of PubM ed [102], whose contents are curated by the $N$ ational Library of M edicine and distributed by the $\mathrm{N}$ ational $\mathrm{C}$ enter for Biotechnology Information, cannot be overstated. D ata from the literature is culled to add value in the form of curated collections, such as the Side Effects of Drugs (SED) annual [8], published in book format.

Electronic 'full-text' databases centered on drugs add relatively modest value over their paper counterparts. An example is SED BASE [103], the electronic equivalent of SED. SEDBASE is indexed by drugs. That is, given a drug, one can access all its reported adverse effects as narrative text. Some other commercial drug databases utilize a fairly detailed data model: notable examples are Cerner Multum's VantageR x [104], of which a freely available subset, the M ultum Lexicon [105], constitutes a valuable public resource, and First DataBank's D rug Information Framework [106]. Such data models support computations that narrative text alone cannot. N otably, software utilizing them can scan a list of medications and report all possible pairs of drug interactions. The latter databases are often redistributed as embedded components of hospital pharmacy or computed physician order entry (CPOE) systems, allowing potentially lifethreatening interactions to be prevented rather than detected after the event.

One must prioritize individual
pharmacogenomics research
results before they can be
integrated usefully with future
software that uses patient
genotype information to assist drug
prescription.

For adverse effects, however, all these systems' data models are currently relatively limited: the effects are categorized by organ system, but within each category, they are still recorded as text. It is often desirable to be able to search by adverse effect rather than by drug. If a patient taking several medications develops a particular adverse effect, the question is whether any of these medications might be responsible. Similarly, for pre-existing conditions that might have been responsible for a problem (e.g., an enlarged prostate predisposing to urinary obstruction, or a $\beta_{2}$ receptor polymorphism that makes asthma patients refractory to $\beta_{2}$-agonist treatment, as reported by Israel and colleagues [9]), one would wish to know whether any of the medications was contributory. Finally, in research settings such as clinical trials of anticancer drugs, when adverse events are observed, the sponsoring agency often asks the clinician-researchers to assess the contribution to the event of the experimental therapy, concomitant medication, and underlying disease. The attribution task can be onerous and knowledge-intensive: researchers need all the electronic help they can get.

Unfortunately, there is no direct way to answer the latter two questions, given the organization of these databases, because the drugs lack indexing by adverse effect or disease condition. With the addition of another dimension to the data - gene variants - these systems are likely to fall further behind with respect to the gap between desired and actual functionality. Currently, the commercial databases do not model gene variants explicitly (for example, VantageRx still categorizes a pharmacogenetics-related adverse event by organ system).

Possible approaches for improving the quality of electronic access to

pharmacogenomic data

There are two issues involved: improving the data model to make it sufficiently robust to support embedded information systems that augment human decision-making, and prioritizing the vast amount of pharmacogenomics information in order to avoid information overload.

Enhancing the data model

While implementing a satisfactory data model that allows robust searches by criteria other than drugsis not a trivial problem, it helps to definethe scope of the problem based upon existing informatics efforts in the areas of biomedical thesauri and ontologies. The proposed enhancements are not limited to pharmacogenomics data, but we emphasize that, with the existing data model limitations, adding support for gene variants alone will not suffice, given the existence of gene-variant/disease interactions, and the fact that gene variants often manifest as adverse effects.

Some basic definitions

Both thesauri and ontologies are databases that hold information about concepts relevant to a 
particular domain. A thesaurus defines individual concepts, synonyms for concepts (for example, 'acetylsalicylic acid' for 'aspirin') and antonyms, if applicable (for example, 'agonists' versus 'antagonists'), as well as hierarchical relationships between specific concepts and more general concepts (for example, between aspirin and non-steroidal anti-inflammatory drugs). The hierarchy need not be strict: a specific concept can be related to more than one general concept. Thus, chlorpromazine is simultaneously a dopamine antagonist, an anticholinergic and a phenothiazine (the last relationship is important in considering specific side effects such as skin and ocular pigmentation).

O ntologies represent refinements of thesauri in that:

- In an ontology, one can segregate concepts into categories (classes), where each class is described by attributes or properties that describe individual concepts belonging to that class. Examples of classes in the real $\mathrm{m}$ of therapeutics include therapeutic agents, diseases/ailments, definitions of adverse effects, and genes and gene variants, both common (polymorphisms) and rare. Examples of attributes include chemical structure, which applies to drugs, or DNA sequence, which applies to genes/gene variants.

- The relationships between concepts or concept classes can be more general (for example, related not only to categorization). Thus, a 'used-in-treatment-of' relationship can exist between individual therapeutic agents and diseases. Such relationships are being modeled in an open-source drug ontology [10] being jointly developed by the $M$ ayo Clinic (MN, USA), the Nashville Veterans Administration Center (TN, USA) and A pelon, Inc. (CT, USA). This is currently in the very early stages of development, and does not yet model adverse effects.

General-purpose support of synonymy is critical. Drugs are often searched via brand names. In the case of adverse effects and diseases, synonyms occur in part because the same terms can have both Anglo-Saxon and G recoLatin equivalents (for example, 'liver failure' and 'hepatic failure', 'vomiting' and 'emesis'). Furthermore, varying grades of the same condition can exist (for example, 'insufficiency' versus 'failure', or nausea as a prodrome/precursor of vomiting), and the taxonomic relationships between these are also significantly more complex than for drugs. Gene variants can be searched via database-specific unique identifiers, by their parent gene, by the protein(s) they encode or the condition(s) they are associated with.

While taxonomic relationships for adverse effects will mostly have to be created de novo by curatorial teams, the situation is better for diseases, where many can be added in bulk from pre-existing taxonomies, such as the Systematic N omenclature of M edicine (SN O M ED ) [107], a thesaurus in widespread production use whose current design closely approximates an ontology.

Prioritizing content: the issues

W hile better modeling will help, it will not solve the problem of providing useful content by itself. We discuss some of the issues by describing a common existing scenario below.

Embedded drug-related information in CPOE systems is used to generate 'alerts' pop-up messages to the prescriber, with the objective of preventing ordering errors. The al erting software is not infallible, for example, it may not communicate in real-time with the clinical laboratory system that has possibly been developed by a different vendor, and so may not be aware of an individual patient's current clinical parameters. Therefore, the software typically allows 'over-riding' of individual alerts. Individual customers/institutions can tune the thresholds for individual alerts, and such tuning is often a fine art: too many alerts of minimal practical consequence have little more than nuisance value. In systems that lack tuning capabilities, or that have been improperly tuned, the volume of nonhelpful alerts can be unacceptably high and a source of physician dissatisfaction with these systems, which have aroused significant controversy with respect to the pros and cons [11]. $\mathrm{N}$ umerous unhelpful alerts can paradoxically increase the human-error rate by increasing 'noise-to-signal' ratio, making already busy physicians more likely to ignore the few genuinely important alerts.

With an additional pharmacogenomics dimension to patient data, there is a real danger that the volume of unhel pful messages may be even higher, if every possible interaction between a patient's gene variants and medications is reported indiscriminately. Clearly, the information must be prioritized and filtered. $\mathrm{H}$ owever, systematic strategies for doing so are not readily apparent, given the limitations of 
the clinical research scenarios discussed earlier. While all agree that an atypical pseudocholinesterase phenotype absolutely contraindicates the use of succinylcholine, in what circumstances should the clinician utilize information on acetylator status? $M$ any difficult decisions must be made, and individual vendors or institutions are not in the best position to make them. As has been the case with clinical guidelines in non-pharmacogenomics settings, consensus comes about only on a case-by-case basis, upon the accumulation of extensive experience.

\section{Acknowledgments}

NIH grants U 01 ES10867 (Nadkarni) and U 01 GM 61388 (Wiepert). The authors would also like to thank Dr Richard Weinshilboum of the M ayo C linic for hisfeedback.

\section{Bibliography}

1. Blum RA, Wylie N, England T, French C: HIV resistance testing in the USA - a model for the application of pharmacogenomics in the clinical setting. Pharmacogenomics 6(2), 169-179 (2005).

2. Afshar M, Rouini M, Ala S: D extromethorphan metabolic phenotyping in an Iranian population. Eur. J. Clin. Pharmacol. 60(12), 849-854 (2005).

3. H erman D, Locatelli I, Grabnar I et al.: Influence of CYP2C 9 polymorphisms, demographic factors and concomitant drug therapy on warfarin metabolism and maintenance dose. Pharmacogenomics]. 5(3), 193-202 (2005).

4. Flores D L, Alvarado I, Wong M L, Licinio J, Flockhart D: Clinical implications of genetic polymorphism of CYP2D 6 in Mexican Americans. Ann. Intern. M ed. 140(11), W 71(2004).

5. Gardiner SJ, Begg EJ: Pharmacogenetic testing for drug metabolizing enzymes: is it happening in practice? Pharmacogenet. Genomics 15(5), 365-369 (2005).
6. M CLeod HL: D rug pathways: moving beyond single gene pharmacogenetics. Pharmacogenomics 5(2), 139-141 (2004).

7. Yengi LG: Systems biology in drug safety and metabolism: integration of microarray, real-time PCR and enzyme approaches. Pharmacogenomics 6(2), 185-192 (2005).

8. Aronson J: Side Effects of D rugs Annual. Elsevier Science Publishing C 0 ., Amsterdam, The N etherlands (2004).

9. Israel E, D razen JM , Liggett SB et al.: Effect of polymorphism of the $\beta(2)$-adrenergic receptor on response to regular use of albuterol in asthma. Int. Arch. Allergy Immunol. 124(1-3), 183-186 (2001).

10. Chute CG, Carter JS, Tuttle M S, H aber,M, Brown $\mathrm{SH}$ : Integrating pharmacokinetics knowledge into a drug ontology: as an extension to support pharmacogenomics. AM IA Annu. Symp. Proc., 170-174 (2003).

11. Berger RG, Kichak JP: Computerized physician order entry: helpful or harmful? J. Am. M ed. Inform. Assoc. 11(2), 100-103 (2004).
Websites

101. www.ncbi.nih.gov/About/primer/pharm.html $\mathrm{N}$ ational Center for Biotechnology Information website: $O$ ne size does not fit all: the promise of pharmacogenomics (Accessed June 9, 2005).

102. www.ncbi.nih.gov/pubmed The PubM ed website (Accessed June 9, 2005).

103. http://library.dialog.com/bluesheets/html/bl0 070.html Thomson/D ialog, SED Base: Side Effects of D rugs website (Accessed June 9, 2005).

104. www.multum.com/VantageR XD B.htm Cerner Corporation, The VantageR $x$ database website (Accessed June 8, 2005).

105. www.multum.com/VantageR XD B.htm Cerner Corporation, M ultum Lexicon website (Accessed June 8, 2005).

106. www.firstdatabank.com/integrated_content/d rug_information/

First D ataBank, D rug Information Framework website (Accessed June 5, 2005).

107. www.snomed.org College of American Pathologists, SN O M ED Clinical Terms (SN O M ED CT) website (Accessed February 10, 2002). 